\title{
Precise calibration of the dependence of surface brightness-colour relations on colour and class for late-type stars ${ }^{\star}$ (Corrigendum)
}

\author{
A. Salsi ${ }^{1}$, N. Nardetto ${ }^{1}$, D. Mourard ${ }^{1}$, O. Creevey ${ }^{1}$, D. Huber ${ }^{2}$, T. R. White ${ }^{3,4,5}$, V. Hocdé ${ }^{1}$, F. Morand ${ }^{1}$, \\ I. Tallon-Bosc ${ }^{6}$, C. D. Farrington ${ }^{7}$, A. Chelli ${ }^{1}$, and G. Duvert ${ }^{8}$ \\ 1 Université Côte d'Azur, OCA, CNRS, Laboratoire Lagrange, France \\ e-mail: anthony.salsi@oca.eu \\ 2 Institute for Astronomy, University of Hawaii, 2680 Woodlawn Drive, Honolulu, HI 96822, USA \\ 3 Sydney Institute for Astronomy, School of Physics A28, The University of Sydney, NSW 2006, Australia \\ 4 Stellar Astrophysics Centre, Department of Physics and Astronomy, Aarhus University, 8000 Aarhus C, Denmark \\ 5 Research School of Astronomy and Astrophysics, Mount Stromlo Observatory, The Australian National University, Canberra, \\ ACT 2611, Australia \\ ${ }^{6}$ Univ. Lyon, Univ. Lyon 1, Ens de Lyon, CNRS, Centre de Recherche Astrophysique de Lyon UMR5574, \\ 69230 Saint-Genis-Laval, France \\ 7 The CHARA Array, Mount Wilson Observatory, Mount Wilson CA91023, USA \\ 8 Univ. Grenoble Alpes, CNRS, IPAG, 38000 Grenoble, France
}

A\&A 640, A2 (2020), https://doi.org/10.1051/0004-6361/202038012

Key words. techniques: interferometric - stars: atmospheres - stars: late-type - stars: fundamental parameters - errata, addenda

In the original paper, Salsi et al. (2020), there is a mistake in the calculation of the uncertainty on the angular diameter estimate. Equation (8) should be written as follows:

$\sigma_{\theta_{\mathrm{LD}} \text { a,bhot }}=2 \ln (10) \theta_{\mathrm{LD}} \sqrt{\sigma_{\theta_{\mathrm{LD} \text { a, }}}^{2}+\sigma_{\theta_{\mathrm{LD}_{\text {phot }}}}^{2}}$.

The photometric part of the uncertainty, $\sigma_{\theta_{\mathrm{LD} \text { phot }}}$ (Eq. (10) in the paper), also contains an error. The correct formula is following:
$\sigma_{\theta_{\mathrm{LD} \text { phot }}}=\sqrt{(0.1+a)^{2} \sigma_{V}^{2}+a^{2} \sigma_{K}^{2}+(0.1+0.881 a)^{2} \sigma_{A_{V}}^{2}}$.

Only considering arbitrary uncertainties of 0.022 mag on both $V$ and $K$ magnitudes leads to a precision of $1.30 \%$ on the angular diameter, and not $1.70 \%$ as indicated in the paper. The conclusions in the paper remain unchanged.

\section{References}

Salsi, A., Nardetto, N., Mourard, D., et al. 2020, A\&A, 640, A2

^ Based on CHARA/VEGA observations. 